\title{
PHYTO-PHARMACEUTICAL DEFINITION UNDER DRUG REGULATIONS, INDIA NEEDS AN URGENT REVIEW
}

Dear Reader,

An Amendment to the Drugs and Cosmetics Regulations to permit scientifically developed plant based leads as drugs named "phytopharmaceuticals", was notified for the first time in November 2015. This regulation has now been further amended and revised to put it under new drug definitions and provided more provisions for greater clarity in 2019. However, having championed these regulations (as part of the Indian Pharmacopoeia Commission's Expert Committee on Herbals) for nearly a decade a greater number of drugs should have come out through this route given the scientific capability of the Indian drug sector and academia. However, the only

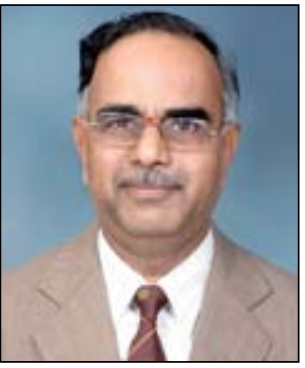
information in the public domain as on date is the approval for conducting human clinical trials of a plant based lead for testing the protective or curative potency of Coculous hirsutus against SARS - CoV-2 given by the Drugs Controller General of India. A purified fraction of the stem of this plant commonly called broom creeper has been tested for sinnococculine markers as the lead drug candidate.

In personal discussions, the sectorpoints out that one of the difficulties to enterphytopharmaceuticals development amongst others, is the way it has been defined. To qualify as a phytopharmaceutical, it has to be "a purified and standard fraction, with defined minimum four bio-active or phyto-chemical compounds (qualitatively and quantitatively assessed), of an extract of a medicinal plant or its part......"

Most scientists point out that the definition is not in line with the global regulatory approach and is highly prescriptive. Recognition that the purified fraction needs to be standardized to demonstrate similarity and uniformity of the batches of the fraction produced is acceptable. However, specifying the number and nature of the marker compounds acts as a stumbling block. While theoretically, 4 markers may be present in the raw botanical, as the commercial process progresses to convert the botanical to an extract, and further processed to a fractionate, which is then formulated as a drug in a dosage form in one or more steps the same 4 markers may not be present or at detectable and quantifiable levels at each stage. The definition of a phytopharmaceutical to be a purified and standard fraction was put in the regulation historically to differentiate from one step extracts (aqueous, alcoholic, aq-alcoholic) which are in the domain of Ayurvedic drugs in the regulations.

Globally the botanical concentrates or fractions need to be characterized chemically and the methods used to demonstrate similarity / uniformity of the batches produced is to be described in the applications. The nature and number of markers etc. of such quality examination / specifications are left to the applicant which is appropriate science. The document "Guidance to Industry for Botanicals as Drugs", of the United States Food and Drug Administration (USFDA) specifies this approach. The 2 botanical drugs which have been given marketing authorization so far follows such a review and does not insist on the number of markers. The botanical drug Veregen, an ointment for genital warts that is derived from green tea leaves, was standardized to sinecatechins. The second botanical drug is Fulyzaq, which is made from the red sap of the Croton lechleri plant, a South American tree referred to as the dragon's blood tree, standardized to crofelemer. This is for the treatment of diarrhea in HIVIAIDS patients who are on anti-retroviral therapy. An exhaustive review of the approach of testing for marker compounds, the nature and number of markers tested, relevance and relation to the efficacy based on study of all the monographs in European Pharmacopoeia (EP) has been published in 2018. This review by Reinhard Länger, and others published in Planta Medica, a reputed peer reviewed journal is titled "Quality Standards for Herbal Drugs and Herbal 
Drug Preparations - Appropriate or Improvements Necessary?". This review underscores that most markers tested were analytical markers and not necessarily responsible for biological activity. Most commonly 2 markers have been prescribed for quantitative testing in EP in a large number of plants / extracts under the test for assay. In the Herbal Medicine Compendium of the USP too, most plant extracts are standardized to a single marker compound.

Narayana et al.: Journal of AOAC International Vol. 102, No. x, 2019, titled,

"A Perspective on Additional Approaches Beyond Organic Marker Compounds While Developing Analytical Monographs for Botanicals" showed that additional approaches are coming up in identifying the nature of the marker compounds. Other compounds such as Primary metabolites, minerals, starches, amino acids etc also contribute to the quality, safety and efficacy of the botanicals.

In view of the above and to provide for a globally acceptable science based definition, the definition of phytopharmaceuticals under the Drugs and Cosmetics Rules, India needs an urgent review. The definition while demanding that adequate scientific testing be adopted by the applicant to build similarity / uniformity in batches of the lead fraction, the method of doing so may be left to the botanical drug developer. The committee of experts reviewing applications would apply varying scientific yardsticks during the review process.

There is hence an urgent need to look at amending the definition of the Phytopharmaceuticals in the Drug regulations without diluting the assessment and control of quality of them. Would the office of Drug Controller General of India, and the concerned officials in the Ministry of Health \& FW, and Department of Pharma initiate action?

D B Anantha Narayana,

https://doi.org/10.53879/id.57.12.p0005

CSO, Ayurvidye Trust. Bangalore

\section{About The Guest Editor}

Dr. Anantha Narayana, is the Chief Scientific Officer, AYURVIDYE TRUST, Bangalore. He Championed the Notifications of Supplements and Nutraceuticals Regulations, FSSAI, 2016 Updated in 2017 and Phytopharmaceuticals as Drugs under Drugs \& Cosmetics Act \& rules, 2016. He is a recipient of Indian Drugs award for Contribution to IDMA and Indian Drugs and is a recipient of Eminent Pharmacist's Award of IPA, 2007. Currently is an expert member, amongst others contributing significantly to 1) Member-Expert committee - NonSpecified Foods \& Food Ingredients - FSSAI, 2) Chairman-Expert Committee-Advertisement \& Claims - FSSAI, 3) Chairman-Scientific Panel - Nutraceuticals of FSSAI. 4) Chairman - Phytopharmaceuticals \& Herbal products of Indian Pharmacopeia Commission 5) Member-Steering Committee of NMPB, Ministry of Ayush.

He continues to guide youngsters in research and also guides many startup firms in the area of Supplements/ Nutraceuticals, Foods, herbals and cosmetics.

$\square$

If you would like to comment on the editorial please write to us at publications@idmaindia.com 